\title{
Nefrosis Lipoidea y alergia por alimentos
}

\author{
Dr. Francisep Díaz H.* Dra. Eda Lagomarsino** Int. Lałis Marín*** Int. Ignacio Navarrete***
}

\begin{abstract}
A total of twenty children with a diaguosis of prymery nephrosis were randonly chosen from the Nephrolor gy Unit of the Area Sur-Oriente of Santiago de Chile. Ages ranged between 1 and 11 years. Diaynostic criteria of nephrosis was; al Albuminaria greater than $100 \mathrm{mgs} / \mathrm{kg} / 24 \mathrm{~h}$ : bj Hipoalbuminenia, of protein electrophoresis with an increase in the alpha 2 traxtion, and a compatible renal biopsia.

dil patients had an Inmunological Prophile pertamied, which measured celular response (PPD \& DNCB!. humoral resputise (fkbrile aghutinins \& Schick test) and intradermal teasting axainst 3 fords frecuently ingested in Clible. Ten patients were randomly hospitalized and elimination diets pertomed during nise days. On the tenth day an induction test was perforned. Samples for albuminuria, leucocyte counts and absolute eosinuphiles detenninatiun were ubtained at $1,2,6,24,7 \mathrm{~L}$ hours after the induction test was pertirmed.

We observed that an allergyc tamily history predominated whes compaired with a renal disease famsly background. diminished celular ressymse and low sensibilizing potentiad proteins sensitize these patients, who when subjected to the elimination diets, have remisinn of their nephrosis or (at reduce the therapeutic steroid? dostes. We comclude that patient's specilic protems sensibility wat influence the clinical crsurse of lipuid nephrosis.
\end{abstract}

En 1916 Munk por primera vez llama Vefrosis Lipoidea a una entidad caracterizada por mortología glomerular normal y la presencia de inclusiones grasas en los túbulos renales. Posteriormente, se descarta la hipótesis de que la enfermedad es una alternación del metabolismo lipídico, proponiéndose, en los siguientes 75 años, una serie de denominaciones alternativas. (Ej.: Síndrome de proteinutia, nefropatias a cambios minimos, enfermedad de la célula epitelial glomerular, etc.), en un afán de describirla desdé el punto de vista clínico e histopatológico.

La práctica corriente de la biopsia renal, microscopía electrónica e inmunofluorescencia, permitieron demostrar que esta entermedad puede tener aspectos histológicos variables. Actualmente, se prefiere el término de Síndrome Nefrótico $(\mathrm{SN})^{2}$

EI SN se define por un conjunto de síntomas que implican: edema, proteinuria importante $y$ perturbaciones protidolipídicas que asocian hipoproteinemia con alteraciones electroforéticas (descenso de la albúmina y elevación de $\mathrm{Alf} \mathrm{a}_{2}$ globulinas) y un aumento de los lípidos séricos. ${ }^{3}$

EI SN puede ocurrir en el curso de varias enfermedades glomerulares, ${ }^{4}$ incluso en la glomerulonefritis aguda, ${ }^{5}$ y además con enfermedades sistemáticas como: Lupus eritematoso diseminado, púrpura anafilactoideo, trombosis de la vena renal, amiloidosis y diabetes mellitus.

\footnotetext{
*Dr. Francisco Diaz H. Jefe Unidad Alergia Hospital Sótero del Rio, Santiago de Chile.

**Dra. Eda Lagomarsino. Jefe Nelrología Intantil Hospital Jasefina Martínez de Ferrari. Santiago de Chile.

***Intemos Medicina Pontificia Universidad Catúlica de Chile.
}

En los nin̄os, estas causas secundarias representan menos del $10 \%$ de todos los síndromes nefróticos ${ }^{6}$ correspondiendo el $\$() \%$ restante a Nefrosis Lipoidea. La incidencia de la nefrosis lipoidea (NL) es 15 veces inayor en el niño que en el adulto, con su mayor incidencia entre 1 y 3 años de vida, siendo relativamente raro el inicio después de los 10 añus.

La etiología de NL es desconocida. Ocasionalmente se han descrito recaidas de $\mathrm{NL}$, en relación a alergias alimentarias? Las atopias son más tiècuentes en las NL que en niños sanos." En la Universidad de Miami está en plena etapa de evaluación la relación entre NL y la hipersensibilidad a alimentos.

Por ser la alergia por alimentos de alta frecuencia en la infancia, ${ }^{10,11}$ nos motivamos a buscar una asociación etiopatogénica entre ésta y la NL. Asimisno, estudiar la capacidad de respuesta inmunitaria humoral y celular en nuestros pacientes, por haberse comunicado alteraciones en dicho rubro. ${ }^{12,13}$

Otro hecho que apoya esta etiopatogenia comun, es que las NL asnciadas a otras alergias presentan recaídas precipitadas por cuadros de rinitis alérgicas. ${ }^{14}$ Todo esto hace suponer que algún tipo de alergia se halla involucrada en la génesis de la NL..$^{15}$

\section{MATERIAL Y METODOS}

Se estudian 20 pacientes nefrósicos primitivos, escogidos al azar en el Servicio de Nefrología Infantil del Area Sur-Oriente de Santiago de Chile, cuyas edades fluctúan de $\mathrm{I}$ a $\mathrm{Il}$ años. 
Se usó como criterio diagnóstico de Nefrosis Lipoidea:

a) Albuminuria mayor de $100 \mathrm{mg} / \mathrm{kg}$ en 24 horas.

b) Hipoproteinemia

c) Electrofóresis de Proteínas con baja de la albúmina y aumento de la fracción Alta $y$

d) Edema.

Complementados con Biopsia Renal (6 pacientes), según criterios diagnósticas anatomopatológicos de Rosemberg et al. ${ }^{16}$ Recopilados los antecedentes personales y/o familiares de manifesta-

ciones alérgicas o renales, fueron sometidos a un perfil inmunológico, detectándose capacidad de respuesta inmunitaria celular (PPD y DNCB) y capacidad de respuesta humoral con Reaccicnes de Aglutinación medidas antes de 15 dias después de $I$ dosis de vacuna antitífica, reacción de Schick e intradermorreacciones a trece alimentos de uso frecuente en nuestra dieta nacional.

El PPD se consideró positivo sobre $5 \mathrm{~mm}$ y el DNCB (dinitroclorobenceno) cuando hubo una diferencia de más de dos cruces entre la dosis sensibilizante y la que mide respuesta celular, de manera de obviar la dermitis por DNCB que da falsos positivos. Cabe mencionar que todos los niños habian recibido vacuna $B C G$, según programa del SNS, asimismo la antidiftérica.

El Schick fue índice de buena respuesta humo. ral cuando la reacción era negativa, y las intradermorreacciones con extractos de diagnóstico de alimentos se consideraron positivas cuando daban dos a cuatro cruces respecto al control.

Al azar se hospitaliza a 10 pacientes del gnupo estudio y se les elimina de la dieta aquellos alimentos a los cuales fueron cutáneo sensibles. Al $10 .^{\circ}$ día se realiza una prueba de provocación, según método de Rowe modificado, ${ }^{17},{ }^{18}$ administrándose los alimentos a los que fueron más sensibles, en el doble de la cantidad habitual, en forma. pura y en ayunas. Previo a esto, se tomaron muestras para cuantificar albuminuria, leucocitos y recuento absoluto de eosinófilos en sangre periférica, estudiando sus variaciones a la hora, dos horas, seis, veinticuatro y setenta y dos horas de la provocación.

\section{RESUltaLos}

De los 20 niños, 10 eran mujeres y 10 hombres.

La edad de comienzo de la NL fue entre 1 y 3 años en $80 \%$ de los casos. La aparición más tardía fue a los 9 años 8 meses. La proteinuria en mgs/kg/24 h. varió de un mírimo de 105 a un máximo de 700 , siendo la media de $366 \mathrm{mg} / \mathrm{kg} / 24 \mathrm{~h}$.

Los valores de proteínas plasmáticas variaron de 2,98 a $5,3 \mathrm{~g} \%$, con una media de $4,04 \mathrm{~g} \%$

La electrofóresis de proteinas presentó en todos ellos una baja de la albúmina (valores entre $13,2 \%$ a $88,9 \%$ ) con un aumento de la fracción $\mathrm{Alfa}_{2}$ (con valores entre $25,3 \%$ a $47,7 \%$ ).

El colesterol, elevado, tuvo valores entre $370 y$ $860 \mathrm{mg} \%$.

El complemento fue normal en todos los pacientes en que se midió (nueve), excepto en uno.

Del punto de vista clínico, todos hicieron edemas y mantuvieron cifras tensionales nomales. Hematuria se presentó en 2 casos.

19 pacientes fueron corticoidesensibles y uno corticoidedependiente, necesitando en dos oportunidades tratarniento inmunosupresor asociado, previo a nuestro estudio.

$\mathrm{La}$ biopsia renal en los casos que se realizó (25\%) dio alteraciones minimas glomerulares.

Se han controlado entre 3 meses a 60 meses, con una media de 18 meses. El mínimo de recaídas previo al estudio varió de 0 a ocho, con una media de 1,5 recaídas.

Lo antedicho representa el análisis nefrológico, que se expresa en la Fig. 1.

Desde el punto de vista alérgico, el $75 \%$ presentó antecedentes atópicos personales y un $30 \%$ relató antecedentes atópicos familiares. No hubo antecedentes de nefropatías tamiliares.

El PPD fue negativo en $70 \%$ y el DNCB en un $80 \%$ de los casos. Las reacciones de aglutinación dieron resultados positivos en un $85 \%$, Schick (-) en un $100 \%$ y las pruebas cutáneas positivas en un 100\%. (Fig. 2)

Los alimentos más sensibilizantes resultaron ser los siguientes: Leche de vaca y came de vacuno $65 \%$, (Fig. 3)

La provocación alimentaria fue acompañada de la aparición de síntomas alérgicos en un $90 \%$ de los casos, siendo los más frecuentes: prurito, eritema facial, rash urticarial, nausea o vómito y en 9 de los 10 casos se observó poliuria inmediata.

Comprobación hematológica ${ }^{18,23}$ se obtuvo en 
Figura N." 1

Análisis nefrológico de los pacientes estudiados.

\begin{tabular}{|c|c|c|c|c|c|c|c|}
\hline $\begin{array}{l}\text { Caso } \\
N .^{\circ}\end{array}$ & Sexo & Edad inicio & $\begin{array}{c}\text { Proteinuria } \\
\text { Lláxima } \\
\text { mg/kg/24 hrs. }\end{array}$ & $\begin{array}{c}\text { Proteínas } \\
\text { Plasmáticas } \\
\text { gn } \%\end{array}$ & $\begin{array}{l}\text { Elect } \\
\text { Alb. } \\
\%\end{array}$ & $\begin{array}{c}\text { oresis } \\
\mathrm{Alfa}_{2} \\
\%\end{array}$ & $\begin{array}{c}\text { Colesterol } \\
\text { Ing. } \%\end{array}$ \\
\hline 1 & $\mathbf{F}$ & $1 \mathrm{a} 7 \mathrm{~m}$ & 480 & 4,65 & 34,0 & 28,3 & 458 \\
\hline 2 & $\mathbf{M}$ & $6 a$ & 170 & 4,90 & 38,9 & 25,3 & 450 \\
\hline 3 & $\mathbf{M}$ & $1 \mathrm{a} 6 \mathrm{~m}$ & 410 & 4,27 & 26,0 & 33,5 & 666 \\
\hline 4 & $M$ & $1 \mathrm{a} 1 \mathrm{Im}$ & 430 & 3,30 & 30,2 & 29,5 & 550 \\
\hline 5 & $\mathbf{F}$ & 1 a $3 \mathrm{~m}$ & 180 & 4,98 & 21,6 & 38,0 & 418 \\
\hline 6 & $\mathbf{F}$ & 2 a $5 \mathrm{~m}$ & 366 & 4,04 & 27,3 & 31,5 & 550 \\
\hline 7 & $\mathbf{M}$ & $1 \mathrm{a} 6 \mathrm{~m}$ & 105 & 5,30 & 36,9 & 26,7 & 470 \\
\hline 8 & $\mathbf{F}$ & 3 a $8 \mathrm{~m}$ & 310 & 3,60 & 23,7 & 37,2 & 660 \\
\hline 9 & $\mathrm{~F}$ & $2 a 7 m$ & 370 & 3,30 & 13,2 & 37,5 & 860 \\
\hline 10 & $\mathrm{~F}$ & $1 \mathrm{a} 5 \mathrm{~m}$ & 450 & 4,46 & 25,3 & 35,2 & 530 \\
\hline 11 & $\mathbf{M}$ & 2 a $11 \mathrm{~m}$ & 350 & 3,90 & 22,7 & 47,7 & 418 \\
\hline 12 & $F$ & $1 \mathrm{a} 5 \mathrm{~m}$ & 590 & 3,65 & 23,0 & 45,2 & 604 \\
\hline 13 & $\mathbf{M}$ & 2 a $3 \pi$ & 320 & 3,65 & $I 6,3$ & 41,8 & 370 \\
\hline 14 & $M$ & 6 a $7 \mathrm{~m}$ & 510 & 4,100 & 25,4 & 33,5 & 460 \\
\hline 15 & $F$ & $2 \mathrm{a} 2 \pi$ & 320 & 4,46 & 28,3 & 35,2 & 550 \\
\hline 16 & $M$ & 5 a $5 \mathrm{~m}$ & 110 & 3,60 & 23,7 & 37,2 & 660 \\
\hline 17 & $M$ & $2 \mathrm{alm}$ & 700 & 4,32 & 27,5 & 33,5 & 812 \\
\hline 18 & $\mathbf{F}$ & $1 \mathrm{am}$ & 390 & 3,90 & 29,3 & 31,5 & 636 \\
\hline 19 & M & 2 a $1 \mathrm{~m}$ & 364 & 3,3 & 33,4 & 32,3 & 520 \\
\hline 20 & $\mathbf{F}$ & $9 \mathrm{a} 8 \mathrm{~m}$ & 200 & 2,98 & 17,9 & 46,4 & 580 \\
\hline
\end{tabular}

un $90 \%$ de los casos: a) descenso de la cifra postprandial de glóbulos blancos, con un aumento del recuento absoluto de eosinófilos en un $80 \%$ y b) aumento del recuento de glóbulos blancos y simultáneamente de los eosinófilos en un $10 \%$ de los casos, no observándose variaciones hematológicas en el $10 \%$ de los casos.

La albumínuria postprandial fue observada en un $50 \%$ de los nefrósicos estudiados, apareciendo recaida de la enfermedad en un $50 \%$.

El análisis del punto de vista alérgico se expresa en las figuras $2,3, y 4$.

\section{DISCUSION}

El trastomo básico y común a todas las formas de nefrosis es el aumento de la permeabilidad del glomérulo para moléculas de mayor tamaño. Pasan proteínas plasmáticas en grandes cantidades, principalmente albúminas por șu mayor concentración plasmática y menor peso molecular, hecho que produce una inversión del cuociente albúmina/globulina. La pérdida de proteinas se traduce en una baja de la presión coloidosmótica, salida del líquido intravascular al intersticial y por ende 
Figura N."2

Antecedentes de manifestaciones alérgicas personales.

Antecedentes de manifestaciones alërgicas fimiliares.

PPD $-70 \%$

DNCB - 80\%

Reacciones de aglutinación + $85 \%$ Schick - $100 \%$

Figura $N{ }^{0} 3$

$\begin{array}{cr}\text { Pruebas cutaneas }+ & 100 \% \\ \text { Leche de vaca } & 65 \% \\ \text { Carne de vacuno } & 65 \% \\ \text { Pescado } & 50 \% \\ \text { Mariscos } & 45 \% \\ \text { Frutas } & 45 \% \\ \text { Yema de huevo } & 40 \% \\ \text { Clara de huevo } & 30 \% \\ \text { Cereales } & 20 \% \\ \text { Soya } & 20 \% \\ \text { Carne de cerdo } & 15 \% \\ \text { Canne de ave } & 10 \% \\ \text { Legumbres } & 5 \% \\ \text { Verduras } & 5 \%\end{array}$

Figura N. ${ }^{0} 4$

Aparición de manifestuciones alérgicas Comprobación hematológica

$90 \%$

$90 \%$

- Disminución de leucocitos y aumento del recuento de eosinófilos $(80 \%)$

- Ammento de leucritos con aumento simultáneo del recuento de eosinófilos (10\%).

Aparición de albuminuria $\quad 50 \%$

Recuída del Síndrome Nefrósico $50 \%$

edema, la mayor parte de las veces alarmante, repercusión clínica que no guarda relación con lo mínimo del daño observado a nivel renal.

La hiperlipemia del SN es de causa incierta. Todos los componentes lipídicos plasmáticos se encuentran aumentados (Colesterol fosfolípidos y triglicéridos), existiendo una relación inversa entre la concentración de albúmina y lípidos. El fenómeno que conduce al aumento de filtración de la membrana glomerular en la NL no está aclarado.

Existen hechos que apuntarian hacia un problema inmunológico:
- EI SN puede reproducirse con Suero Anti. riñón.

- Estímulos inmunológicos inducen una rẹcaída. Ej.: SN por picada de abeja o por exposición a alérgenos inhalantes.

- Enfermedades anergizantes como el saram. pión hacen remitir cuadro.

- Mejoría parcial o total con corticoides yio con inmunodepresores.

Actualmente, la investigación está orientada al terreno inmunológico, específicamente inmunidad celular. Se ha demostrado la mayor producción de MIF (factor inmovilizador de macrófagos) en estos pacjentes.

No se han descrito complejos inmunes en la membrana basal glomerular del riñón, encontrándose complemento normal.

Se ha planteado la existencia de un clon alterado de linfocitos $\mathbf{T}$, producto de un timo patológico, que produciria una linfotoxina anti-riñón. ${ }^{19}$

Giangiacomo, ${ }^{12}$ comparando concentraciones de inmunoglobulinas en el SN idiopático, en Glomerulonefritis aguda y en niños sanos de la misma edad, descubrió que en la NL la IgG plasmática estaba disminuida, pero la IyM era casi el doble antes, durante y después del tratamiento. Postula que la alteración primaria en la NL consiste en una deficiente producción de IgG y eficiente de IgM por parte de los linfocitos $T$.

Nosotros observamos escasa positividad difusa lineal de IgG en la membrana basal, en la inmunotluorescencia del paciente $\mathrm{N}^{\circ} 10$, único paciente al que se le efectuó la biopsia renal antes del inicio de la terapia esteroidal.

Lewinsky, analizando el plasma de niños con NL en busca de complejos inmunes, observó que pacientes con recaída aumentaban sus niveles de complejos IgG significativamente respecto de los controles ( $\mathbf{p} 0,001$ ) y en los niños con NL en remisión ( 0,001 ). Después de seis semanas de remisión igualaron concentración de controles. Esto sugiere un rol patogénico de los complejos inmunes, desconociéndose su mecanismo.

En nuestros resultados, encontramos una capacidad deficitaria, manifestada en un PPD (-) (70\%) y en un DNCB (-) (80\%) y una capacidad de respuesta humoral satisfactoria, manifestada con reacciones de aglutinación $(+)(85 \%)$ y con reacción de Schick (-) (100\%), como asimismo intradermorreacciones positivas a extractos de algún alimento en un 100\%. La inmunología no explica en su totalidad la reacción alérgica, que para ex- 
presarse requiere la mayor parte de las veces de múltiples factores. Nos llama la atención la elevación de los basófilos en la sangre periférica de nuestros nefrósicos, que nos hace sospechar que en la patagonia del SN pudiera estar involucrado un daño por hipersensibilidad retardada mediada por basófilos. ${ }^{1 !}$

Rara vez los alimentos por sí solos desencadenan manifestaciones alérgicas, actuando generalmente junto a otros alimentos, o requiriendo la presencía de otros antigenos (especialmente inhalantes) para desencadenar el cuadro clínico correspondiente, como también hemos vistu con frecuencia que los alimentos actúan de vehículos de agentes contaminantes como hongos 11,81 y bacterias, los que en sí serían los causantes de la manifestación alérgica atribuida a la ingestión de alimentos.

Nos llama la atención que proteínas tradicionalmente poco alergizantes, como la carne de vacuno y la yema de huevo, ${ }^{22,}$ is sensibilizan a nuestros netrósicos y que la presencia de alimentos como factor de sensibilización alérgica adquere mayor importancia que en grupos similares de población estudiados en nuestro medio. Es asi que nuestro equipo investigador, al estudiar el poder alergizante de la proteina de la soya, ${ }^{1 \times}$ observó en un grupo de personas con antécedentes de haber presentado alguna maniféstación alérgica $(A+)$ y en otro sin antecedentes alérgicos $(A-)$, que la sensibilización a alimentos comparativos fue la siguiente; Fig. 5.

$\begin{array}{lccc} & \mathrm{A}+ & \mathrm{A}- & \mathrm{NL} \\ \text { Pescado } & 29.03 \% & 10 \% & 50 \% \\ \text { Leche de vaca } & 22,58 \% & 10 \% & 65 \% \\ \text { Huevo } & 22,58 \% & 10 \% & 40 \% \\ \text { Came de } & & & \\ \text { vacuno } & 3,22 \% & - & 65 \% \\ \text { Soya } & 16,12 \% & 5 \% & 20 \%\end{array}$

Deducimos de esto la importancia que tiene e] estudio de sensibilización alèrgica a determinadas proteínas antes de indicar una dieta hiperproteica en NL, pues nuestros enfermos al someterse a dietas controladas de eliminación remiten 0 distancian sus crisis, evitando el uso exagerado de corticoides y su asociación a innunosupresores, observando también que cuando el corticoide es empleado se obtiene una respuesta diurética más precoz, asociada a su respectiva dieta controlada de eliminación.

\section{CONCLUSIONES}

Se observó predominio claro de antecedentes alérgicos sobre los antecedentes renales. Aun cuando existia ausencia de antecedentes person:les de alergia, casi todos los pacientes presentan claras manifestaciones alérgicas secuntarias a la ingestión de alimentos. Casi todos evidenciaron una capacidad de respuesta inmunitaria celolar deprimida.

Los alimentos que demostraron ser más sensibilizantes en los nefrósicos fueron: leche de vaca, carne de vacuno, pescado, mariscos, fruta y proteina de soya, en orden decreciente de actividad, hecho importante pues son las principales fuentes proteicas en nuestro medio y las más usadas en las dietas hiperproteicas recomendadas a los pacientes nefrósicos.

Por esto, estimanos cyue la presencia en la dieta de proteínas especificamente alergizantes para catda individuo tiene influencia destavorable en la evolución clínica de la nefrosis lipoidea, haciétdose necesario incorporar este enfocgue alérgico en el trataniento integral de la enfennedad.

\section{REFEREYCLAS}

1 Mank, F. Die Neprosen. Med. Xlin. 12: 1019, 1047 y 1073 , 1916.

${ }^{2}$ Bamett, M.L. Forman, C.S. y Lausona, H.D.: The Nephrotic. Syndrome. Natural History of the Disease. Pediatrics 5: 486, 1950 .

${ }^{3}$ Rower, P., Habib R., Mathieu H., Broyer M., Sindrome Nefrótico. Nefrologia Pediátrica. Ed. Turav. Barcelona, p. 181, 1975.

'Chirg.J. Habib, A., White, H.H.R. Tatholoy of the Nephrotic Syndmone in Children. A report for the International Stady of Kidney Disease in Children. Lauket l: 299. 1970.

5Whisun, S.C.F. y Heymanin, W. Acute Glomerulonephritis with the nephrotic syndrome. Pediatrics $23: 874,1959$.

6habib, R. y Kleinkrech, C. The primary nephrotic syndrome of ehildhood. Classification and Chinicopathology Study of 406 cases in Summers. S.C. (ed). Patholoky Annual (New York: Appleton - Contury - Crofts 1971) p. 417

T.Mutsunura, T, $y$ Karoume', T. The role of allerty in the pathogenesis of the nephrotic syndrome, Jap. J. Pediat. 14: 921. 1961.

'Thomson, P.D., Barret, T.H., Stokes C.R., Turner M.H. y Sonthill, JF.: HLA antigens and atopic features in steroid responsive. Nephrotic syndrome of childhood. Lancet 2: 765. 1976.

"Sandberg D.H., Bernstein D.D., Ms. Instosh R.H., Deling, B., St ratss J. hyperse'ssibitivity to foods and Neghrosis. Strauss J. (Ed.), Pedjatric nephrology. Vol. 2, Mami, Symposia Specialists, p. J91, 1975.

loSsolari, M.A. Alergia alimentaria, Clínica y terapéutica Actas II. Congreso Latinoamericano de Alergología. Ed. Universitaria. Quito, Ecuador; 117, 1967.

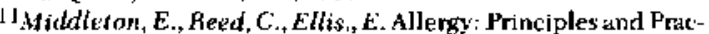
tice the C.V. Mosby Company, St. Louis P. 1401978. 
${ }^{12}$ Giangiacomo, J., Cleary, T.G., Cole, B.R. y otros. Serum immunoglabulins in the nephrotic syndrome. A possible cause of minimal-change nephrotic syndrome. N. Engl. J. Med. 293: 8, 1975.

13 Shathouk $b$, R.J. Pathogenesis; of lipoid nephrosis. A disorder of tell fuction Lancet 2: 356,1974 .

${ }^{14}$ Holt G. Nephrotic Syndrome with pollen hypersensitivity Lancet I. 500, 1959.

${ }^{15}$ Reetes W.R. Cameron J.S. Johansson S.C.O. Ogg. C.S. Peters D.K. y Weller R.O. Seasonal nephrotic syndrome. Descríption and immunologicol findıngs. Clinical Allergy S. 1811875.

${ }^{16}$ Rosemberg. H., Vial, S., Clasificación de las Rev. Méd. Chile, 106: 125, 1978 .

${ }^{17} \mathrm{~F}_{0 u t}$, A.: Elimination diets and the patient allergies. Lea and Fabiger, Philadelphía, 1950.

INDíaz, F, Conzatez, C. Estudio clinico y de Laboratorio de
Alergia por alimentos y Soya. Presentado III Congreso Nacional de Medicina de Chile, 1979.

${ }^{19}$ Eyres, K., Mallick N.P., Taylor, G. Evidence for cell-mediated inmunity to renal antigens in minimal-change nephrotic syndrome. Lancet 29: $1158,1976$.

20 Jeutinsky, R.J., Mallerson, P,N, Barret, w., Soothill, J.F. Circulatinginsune complexos in aterojd responsise nephrotic syndrome, N. Engl. J. Med. 298: 126, 1978.

$2 \mid D i a z, F$. Alergia por alimentos. Manual de Pediatria. Departamento de Pediatria Escuela de Medicina Cniversidad Católicu de Chile, paig. 361, 1979.

22 Sonchez Medina, Mario. Alergia alimentaria en América Lat. na. Actas II Congreso Lutincamericann de Alergologia- Editorial L niversitaria. Quito, Ecuador, 1967, págs. 85 y 112.

${ }^{23}$ Beruemun, H., Hastether, J.; Kaboth, W. y cots. Hematologia Medica Editorial Científica Medica. Barcelona 1974. 